\title{
ENHANCING SPARSITY USING GRADIENTS FOR COMPRESSIVE SENSING
}

\author{
Vishal M. Patel, Glenn R. Easley, Rama Chellappa, and Dennis M. Healy, Jr. \\ University of Maryland, \\ College Park, MD 20742 \\ \{pvishalm@umd,geasley@umd,rama@cfar,dhealy@umd\}.edu
}

\begin{abstract}
In this paper, we propose a reconstruction method that recovers images assumed to have a sparse representation in a gradient domain by using partial measurement samples that are collected in the Fourier domain. A key improvement of this technique is that it makes use of a robust generalized Poisson solver that greatly aids in achieving a significantly improved performance over similar proposed methods. Experiments provided also demonstrate that this new technique is more flexible to work with either random or restricted sampling scenarios better than its competitors.
\end{abstract}

Index Terms - compressive sensing, compressed sensing, GradientOMP

\section{INTRODUCTION}

Reconstruction of imagery from an incomplete set of samples from a Fourier representation is an important goal to improve scanning technologies such as magnetic resonance imaging (MRI) and synthetic aperture radar (SAR) imaging. Solutions to such a problem would allow a significant reduction in collection time and improve the capacity to collect very time sensitive events. For example, an MRI scanner records data in a Fourier representation so methods, capable of reconstructing from such partial samples, would greatly improve medical science and reduce a patient's exposure time.

Many MRI and SAR images can be sparse or compressible in some transform domain such as those from a wavelet, gradient or Fourier transform. Images such as angiograms are inherently sparse in the pixel domain or gradient domain. For instance, if the image is piecewise constant then a gradient representation would only contain nonzero values near boundary positions.

Compressive sensing (CS) theory states that it is possible to recover such sparse images from a small number of random measurements provided the undersampling results in incoherent artifacts in the transform domain and an appropriate nonlinear recovery scheme is used $[1,2]$. More precisely, suppose $x \in \mathbb{R}^{N}$ is $K$-sparse in a basis (or a frame) $\Psi$, so that $x=\Psi x_{0}$, with $\left\|x_{0}\right\|_{0}=K \ll N$, where $\|.\|_{0}$ returns the number of nonzero elements. In the case when $x$ is compressible in $\Psi$, it can be well approximated by the best $K$-term representation. Consider a random $M \times N$ measurement matrix $\Phi$ with $M<N$ and assume that $M$ measurements, that make up a vector $y$, are made such that $y=\Phi x=\Phi \Psi x_{0}=\Theta x_{0}$. According to the theory of CS, when $\Theta$ satisfies the restricted isometry property (RIP) [8], we can reconstruct $x$ via its coefficients $x_{0}$ by solving the $\ell^{1}$ minimization problem $[1,2]$

$$
\hat{x_{0}}=\arg \min _{x_{0} \in \mathbb{R}^{N}}\left\|x_{0}\right\|_{1} \quad \text { subject to } y=\Phi \Psi x_{0} .
$$

In the case when $\Phi$ is generated from iid Gaussian distribution and $\Psi$ is a sparsifying basis, then $\Theta$ satisfies the RIP with high probability. Among many other ensembles of random matrices that satisfy the RIP, a partial Fourier matrix where $M$ rows of the $N \times N$ Fourier matrix are selected at random are included.

The optimization problem (1) is often referred to as Basis Pursuit (BP). In the case when there are noisy observations, Basis Pursuit De-Noising (BPDN) can be used to estimate the original image.

Maleh et al. presented in [3] an improved recovery algorithm that can recover sparse gradient images from partial Fourier data using orthogonal matching pursuit (OMP). It is known as the GradientOMP method. The GradientOMP method, however, suffers from some issues in application. In many MRI data collection scenarios, Fourier samples are collected along curves in Fourier space ( $k$-space) and there is usually no reason to discard any samples collected within such curves. This violates GradientOMP's working premise that the sampling set is the union of two radial slices through the origin perpendicular to one another and randomly chosen points in the plane. While this may be approriate for 3D MRI and 2D spectroscopy when the image plane is perpendicular to the collection trajectories, a versitile method should be able to recover imagery from 2D MRI data as well.

In this paper, we present a new method that recovers images that can be sparsely represented by gradients using highly undersampled Fourier measurements. This new 
method is less restrictive on sampling constraints than the GradientOMP and is more robust in the estimation process. A key element in the improvement is that besides using a different/better nonlinear estimator to estimate the gradient field, we solve a generalized Poisson equation to obtain the estimated image. Such a Poisson based solution [4] is much more robust to imperfections in the estimate of the gradients and does not require any constraint on the type of data collections that can be made.

\section{BACKGROUND}

Let $x \in \mathbb{C}^{l \times l}$ and $\Omega$ be some randomly chosen set of frequencies of size $|\Omega|=M$ and $W_{\Omega}: \mathbb{C}^{l \times l} \rightarrow \mathbb{C}^{M}$ to denote the partial Fourier transform operator restricted to $\Omega$. The total-variation (TV) operator is defined as: $T V(x)_{n, m}=$ $\sqrt{p(n, m)^{2}+q(n, m)^{2}}$ where $p$ and $q$ are the discrete gradients of an image $x$, i.e. $p(n, m)=x(n, m+1)-x(n, m)$ and $q(n, m)=x(n+1, m)-x(n, m)$ for $0 \leq m, n \leq l-1$. Given the assumption that $x$ has sparse gradients; that is $\|T V(x)\|_{0}=K \ll N=l^{2}$, and $Y=W_{\Omega} x \in \mathbb{C}^{M}$ (representing the partial Fourier measurements of $x$ ), the general problem is to estimate $x$. It was shown in [2] that for an overwhelming percentage of sets $\Omega$ with cardinality obeying $|\Omega|=M \geq C K \log (N)$, the minimizer to

$$
x_{r e c}=\arg \min _{x^{\prime} \in \mathbb{C}^{N}}\left\|T V\left(x^{\prime}\right)\right\|_{1} \quad \text { s. t. } W_{\Omega} x^{\prime}=W_{\Omega} x
$$

is unique and recovers $x$.

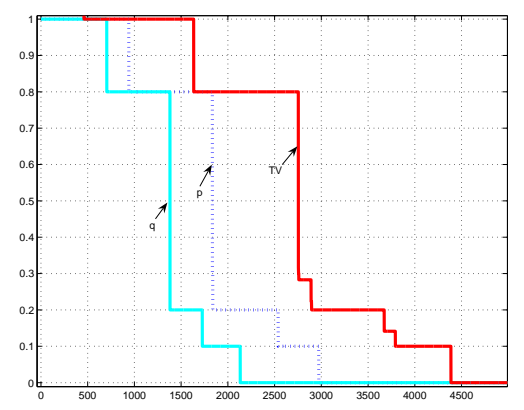

Figure 1: The magnitude of TV (red), $p$ (dotted blue) and $q$ (cyan) coefficients in decreasing order for the Shepp-Logan Phantom image (see Figure 2(a)).

In this paper, we show that instead of reconstructing an image by the $T V$ minimization, one can reconstruct the image by separately reconstructing the gradients and then solving for the image. This allows one to reconstruct the image with a far fewer number of measurements than required by the $T V$ minimization method (see Figure 2). Figure 1 presents an important comparison in the sparsity of $p, q$ and the TV measure. The plots of the sorted absolute values of the coefficients of the gradients $p, q$ and the $T V$ measure for the Shepp-Logan Phantom image indicate that $p$ and $q$ decay much faster than the $T V$ measure. In fact, it is easy to see from the expression of $T V$ that the coefficients of $p$ and $q$ will always decay faster than the coefficients of $T V$. This means our method can take advantage of this and be able to reconstruct an image with far fewer measurements than that required by using the $T V$-based method. Note the assumption that the image of interest has sparse gradients with the use of the TV constraint for estimation has been suggested before $[2,5,6,7]$.

\section{IMAGE GRADIENT ESTIMATION}

By using the properties of the Fourier transform, we can directly express the partial gradients $P=W_{\Omega} p$ and $Q=$ $W_{\Omega} q$, in the Fourier domain from $Y$ as

$$
P=\left(e^{\frac{j 2 \pi k_{2}}{l}}-1\right) Y \text { and } Q=\left(e^{\frac{j 2 \pi k_{1}}{l}}-1\right) Y
$$

where $\left(k_{1}, k_{2}\right) \in \Omega$. Taking into account the presence of additive noise during the measurement process, gradients can be reconstructed by solving the following two constrained optimization problems:

$$
\begin{aligned}
& p_{\text {rec }}=\arg \min _{p^{\prime}}\left\|p^{\prime}\right\|_{1} \text { s. t. }\left\|W_{\Omega} p^{\prime}-P\right\|_{2} \leq \varepsilon \\
& q_{r e c}=\arg \min _{q^{\prime}}\left\|q^{\prime}\right\|_{1} \text { s. t. }\left\|W_{\Omega} q^{\prime}-Q\right\|_{2} \leq \varepsilon
\end{aligned}
$$

where $\varepsilon$ is a noise statistic that controls the fidelity of the reconstruction. It can be shown that the solutions to (4) and (5) will recover the unknown sparse gradients $p$ and $q$ with an error proportional to the noise level. That is, $\| p_{\text {rec }}-$ $p \|_{2} \leq C \varepsilon$, and $\left\|q_{r e c}-q\right\|_{2} \leq C \varepsilon$, where the constant $C$ depends on the restricted isometry constant [8].

There has been a number of approaches suggested for solving BPDN problems such as (4) and (5). In our approach, we shall employ a highly efficient algorithm that is suitable for large scale applications known as the spectral projected gradient (SPGL1) algorithm [9]. See [9] and [10] for the efficiency of spectral projected gradient algorithms compared to OMP.

\section{IMAGE RECONSTRUCTION FROM GRADIENTS}

Once the gradients have been estimated, the problem is to obtain the desired image reconstruction. The gradient field $(p, q)$ of a scalar surface or an image should be integrable (conservative). That is, the integral along any closed curve should be equal to zero. However, this is often not the case when inherent noise during the estimation process contaminates the gradient field.

One of the ways of enforcing integrability is by using the Simchony, Chellappa and Shao (SCS) method [11]. It can be described as follows $[4,11]$. Let $(p(n, m), q(n, m))$ be the estimated non-integrable gradient field. Define the curl and divergence operators as: $C_{p, q}=\operatorname{curl}(p, q)=$ 


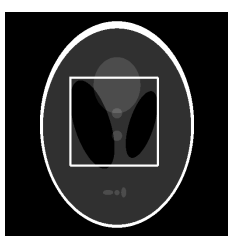

(a)

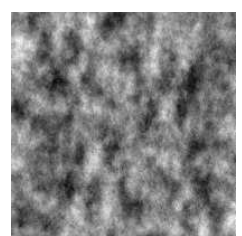

(b)

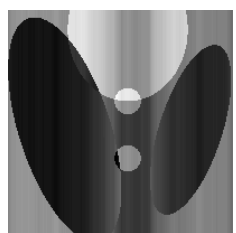

(c)

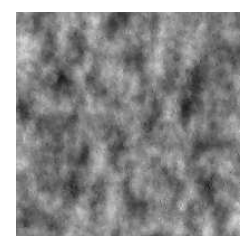

(d)

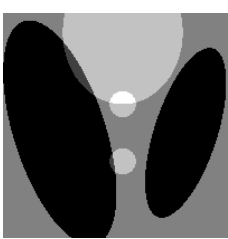

(e)

Figure 2: (a) $512 \times 512$ Shepp-Logan Phantom example. Reconstructed by (b) zero-filling, (c) GradientOMP, (d) TV method, and (e) our proposed method.

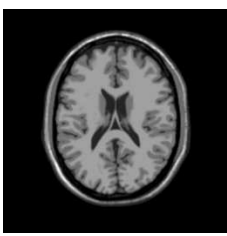

(a)

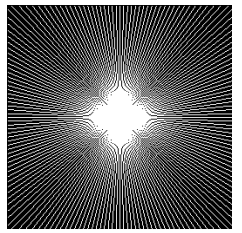

(b)

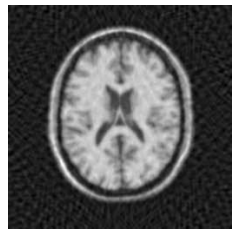

(c)

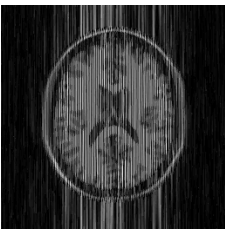

(d)

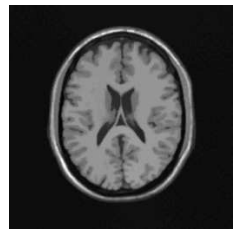

(e)

Figure 3: (a) Original MRI image. (b) Radial $k$-space trajectories. Reconstructed by (c) zero-filling, (d) GradientOMP, and (e) our method.

$\frac{\partial p}{\partial n}-\frac{\partial q}{\partial m}$ and $D_{p, q}=\operatorname{div}(p, q)=\frac{\partial p}{\partial m}+\frac{\partial q}{\partial n}$. The objective is to obtain a surface $\hat{x}(m, n)$ estimate (reconstructed image) such that its gradient field $(\hat{p}, \hat{q})$ satisfies the integrability constraint $C_{\hat{p}, \hat{q}}=0$ and minimizes $J(\hat{x})=\iint[(\hat{p}-$ $\left.p)^{2}+(\hat{q}-q)^{2}\right] d m d n$. The associated Euler-Lagrange equation gives the Poisson equation $\nabla^{2} \hat{x}=\operatorname{div}(p, q)$, where $\nabla^{2}=\frac{\partial^{2}}{\partial n^{2}}+\frac{\partial^{2}}{\partial m^{2}}$ is the Laplacian operator.

Since the Least Square based approaches, such as the one described above, are known not to be robust to noise and outliers, they result in a loss of salient features in the image. Thus, we incorporate in our proposed algorithm an improved Poisson based approach known as the affine transformation on gradients using diffusion tensors [4].

\subsection{Affine Transformation of Gradients using Diffusion Tensors}

Motivated by the application of anisotropic diffusion in image enhancement and denoising, a generalization of divergence based equation for image reconstruction has been suggested [4]. Given that

$$
D(n, m)=\left[\begin{array}{ll}
d_{11}(n, m) & d_{12}(n, m) \\
d_{21}(n, m) & d_{22}(n, m)
\end{array}\right]
$$

be a $2 \times 2$ symmetric positive-definite matrix associated to each pixel location, a generalized relation is then

$$
\operatorname{div}\left(D\left[\begin{array}{c}
\hat{p} \\
\hat{q}
\end{array}\right]\right)=\operatorname{div}\left(D\left[\begin{array}{l}
p \\
q
\end{array}\right]\right)
$$

This equation is the associated Euler-Lagrange equation of the functional

$$
\begin{array}{r}
J(\hat{x})=\iint d_{11}(\hat{p}-p)^{2}+ \\
\left(d_{12}+d_{21}\right)(\hat{p}-p)(\hat{q}-q) \\
+d_{22}(\hat{q}-q)^{2} d m d n .
\end{array}
$$

Let $x_{D}$ denote the lexicographically ordered column vector of $\operatorname{div}\left(d_{11} p+d_{12} q, d_{21} p+d_{22} q\right)$, then the general Poisson equation (an alternative formulation of the general relation) can be simply expressed as $\nabla_{D}^{2} \hat{x}=x_{D}$, where $\nabla_{D}^{2}$ is the weighted Laplacian kernel based on the diffusion tensor $D$ (see [4] for more details). The image can then be efficiently recovered as $\hat{x}=L_{D}^{-1} x_{D}$, where the matrix $L_{D}$ is the sparse weighted Laplacian matrix [4]. Note that this method does not require any restriction on the amount of information gleaned from a partially sampled Fourier data set.

\section{EXPERIMENTS}

In this section, we give some numerical examples to illustrate the performance of our algorithm and compare it with the GradientOMP [3], the $T V$ minimization method [12], and the minimum energy reconstruction by zero-filling [5]. In the first example, we reconstructed a $512 \times 512$ SheppLogan phantom image after $94.6 \%$ random undersampling. As can be seen from Figure 2, the reconstruction by zerofilling, GradientOMP and the $T V$ minimization fail to reconstruct the image perfectly, while our proposed algorithm reconstructs the image exactly $\left(\mathrm{MSE}=3.2 e^{-6}\right)$.

In the second example, Figure 3, we show the reconstruction of a $256 \times 256$ MRI of the brain after $68.5 \%$ undersampling using Cartesian radial sampling. Both Gradien- 
tOMP and zero-filling provide poor image reconstructions, while our method performs significantly better.

Finally, to compare our algorithm in terms of the robustness to noise suppression, we tested a $128 \times 128$ SheppLogan phantom image with additive white Gaussian noise with signal-to-noise ratio of $20 \mathrm{~dB}$. Only $35 \%$ of the Fourier coefficients were used. As can be seen from the Figure 4, reconstruction by the GradientOMP suffers from the vertical streaking artifacts while our method removes these artifacts and provides more improved reconstruction.

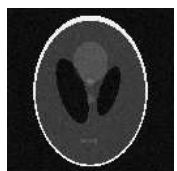

(a)

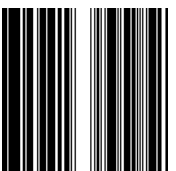

(b)

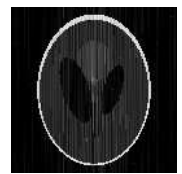

(c)

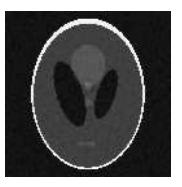

(d)
Figure 4: (a) Noisy $128 \times 128$ phantom image (SNR 20dB). (b) Sampling pattern. Reconstructed image by (c) GradientOMP and (c) by our method.

Remark: Experiments have shown that image reconstruction by the GradientOMP always suffers from the vertical streaking artifacts regardless of the type of sampling being used. Reconstruction by the $T V$ method is effective when the measurements are given along radial lines, however it fails miserably when the coefficients are chosen randomly [3]. Our method is robust to work with either random or restricted sampling and provides much better reconstruction from fewer measurements than the $T V$ method or the GradientOMP.

Recently, there has been a great interest in using $\ell_{p}$ minimization with $p<1$ for compressive sensing [7, 13]. It has been observed that the minimization of such a nonconvex problem leads to recovery of signals that are much less sparse than required by the traditional methods [7]. Our method is flexible enough that, instead of solving problems (4) and (5) using SPGL1, the gradients can be reconstructed by using any of these nonconvex algorithms. It is also possible to reconstruct the gradients from even fewer measurements by adapting the reweighted $\ell_{1}$ minimization algorithm [14]. OMP can also be used to recover the gradients. In this case, our method can be viewed as an improved GradientOMP.

\section{CONCLUSION}

We have proposed a new method of recovering an image when partially sampled data is collected in the Fourier domain and the image is considered to be sparsely represented by gradients. This entails using a robust general Possion solver to obtain the reconstructed image. Various experiments have shown a great improvement in quality of reconstruction as well as a robustness to various sampling scenarios over similar type schemes.

\section{REFERENCES}

[1] D. Donoho, "Compressed sensing," IEEE Trans. Info. Theory, vol. 52, no. 4, pp. 1289-1306, April 2006.

[2] E. Candes, J. Romberg and T. Tao, "Robust Uncertainty Principles: Exact Signal Reconstruction from Highly incomplete Frequency Information," IEEE Trans. Info. Theory, vol. 52, no. 2, pp. 489509, Feb. 2006.

[3] R. Maleh, A. C. Gilbert, and M. J. Strauss, "Sparse Gradient Image Reconstruction Done Faster", Proc. ICIP, vol. 2, pp. 77-80, Oct. 2007.

[4] A. Agrawal, R. Raskar, and R. Chellappa, "What is the Range of Surface Reconstruction from a Gradient Field?", Proc. ECCV, May 2006.

[5] M. Lustig, D. Donoho, and J. M. Pauly, "Sparse MRI: The Application of Compressed Sensing for Rapid MR Imaging," Magnetic Resonance in Medicine,vol. 58, no. 6, pp. 1182-1195, Dec. 2007.

[6] E. Y. Sidky, C.-M. Kao, and X. Pan, "Accurate image reconstruction from few-views and limited-angle data in divergent-beam CT,” J. X-ray Sci. Tech., vol. 14, pp. 119-139, 2006.

[7] R. Chartrand, "Nonconvex compressive sensing and reconstruction of gradient-sparse images: random vs. tomographic Fourier sampling", ICIP, Oct. 12-15 2008.

[8] E. Candes, J. Romberg and T. Tao, "Satble signal recovery from incomplete and inaccurate measurements," Communications in Pure and Applied Mathematics, vol. 59, pp. 1207-1223, 2006.

[9] E. van den Berg and M. P. Friedlander, "SPGL1: A solver for large-scale sparse reconstruction", http://www.cs.ubc.ca/labs/scl/spgl1/.

[10] M. Figueiredo, R. Nowak, and S. Wright, "Gradient projection for sparse reconstruction: Application to compressed sensing and other inverse problems", IEEE Journal of Selected Topics in Sig. Proc., vol. 1, no. 4, pp. 586-597, Dec. 2007.

[11] T. Simchony, R. Chellappa, and M. Shao, "Direct analytical methods for solving Poisson equations in computer vision problems. , " IEEE Trans. Pattern Anal. Machine Intell., vol. 12, no. 5, pp. 435-446, May 1990.

[12] J. Romberg, L1-Magic, http://www.acm.caltech.edu/11magic/

[13] R. Chartrand, "Exact reconstructions of sparse signals via nonconvex minimization", IEEE Signal Process. Lett., vol. 14, pp. 707$710,2007$.

[14] E. J. Candes, M. Wakin and S. Boyd, "Enhancing sparsity by reweighted $\ell_{1}$ minimization", J. Fourier Anal. Appl., vol. 14, pp. 877-905, 2008.

[15] E. van den Berg, M. P. Friedlander, G. Hennenfent, F. J. Herrmann, R. Saab, O. Yilmaz "Sparco: A Testing Framework for Sparse Reconstruction," Technical Report TR-2007-20, Department of Computer Science, University of British Columbia, Oct. 2007. 